\title{
Supporting Information for: DART-MS spectral similarity of infrared thermally desorbed solid particulate and solution cast propellant samples
}

\author{
Thomas P. Forbes* and Greg Gillen \\ National Institute of Standards and Technology, Materials Measurement Science Division, \\ Gaithersburg, MD 20899, USA \\ *Corresponding author: E-mail: thomas.forbes@nist.gov, Tel: 1-301-975-2111
}

\section{Supporting Information Table of Contents}

\section{Methods}

Table S1. IRTD-DART-MS system parameters and settings.

SEM-EDS Instrumentation.

\section{Supplemental Figures}

Figure S1. Ion ratios of solid- and liquid-based Triple Seven BPS samples.

Figure S2. SEM-EDS of solid and solution cast Pyrodex BPS samples.

Figure S3. Ion ratios of black powder substitutes and neat potassium perchlorate.

Figure S4. SEM-EDS of solution case potassium perchlorate samples. 


\section{Methods}

Table S1. IRTD-DART-MS system parameters and settings.

\begin{tabular}{|l|l|l|l|}
\hline \multicolumn{4}{|c|}{ IRTD Parameters } \\
\hline Power & $100 \%$ & Emission duration & $15 \mathrm{~s}$ \\
\hline \multicolumn{4}{|c|}{ DART Ion Source Parameters } \\
\hline DART gas & $\mathrm{N}_{2}$ & DART gas temperature & $400{ }^{\circ} \mathrm{C}$ \\
\hline DART grid voltage & $100 \mathrm{~V}$ & Vapur flow rate & $4 \mathrm{~L} / \mathrm{min}$ \\
\hline \multicolumn{4}{|l|}{ AccuTOF Parameters } \\
\hline Orifice 1 voltage & $-20 \mathrm{~V}$ & Orifice 2 voltage & $-5 \mathrm{~V}$ \\
\hline Ring lens voltage & $-5 \mathrm{~V}$ & Peaks voltage & $-400 \mathrm{~V}$ \\
\hline Detector voltage & $-2300 \mathrm{~V}$ & Orifice temperature & $100{ }^{\circ} \mathrm{C}$ \\
\hline m/z range & $\mathrm{m} / \mathrm{z} 30-\mathrm{m} / \mathrm{z} 600$ & & \\
\hline
\end{tabular}

SEM-EDS. Images of solid and solution cast propellant samples were acquired with an FEI Apreo S Scanning Electron Microscope (SEM). The microscope was outfitted with a dual Bruker AXS Inc. silicon drift detectors (SDD) for Energy Dispersive X-Ray Spectroscopy (EDS) microanalysis. SEM images were generally collected with $1 \mathrm{keV} 0.25 \mathrm{pA}$ electron beam and $3 \mathrm{~mm}$ working distance of the in-column T1 backscattere electron detector. EDS mapping used a $10 \mathrm{keV} 0.4 \mathrm{nA}$ electron beam with $10 \mathrm{~mm}$ working distance. Solid particle samples were ground with mortar and pestle and deposited onto carbon GSR mounts by vacuum impaction. Solution cast samples were deposited onto silicon wafers and allowed to air dry prior to analysis.

\section{Supplemental Figures}

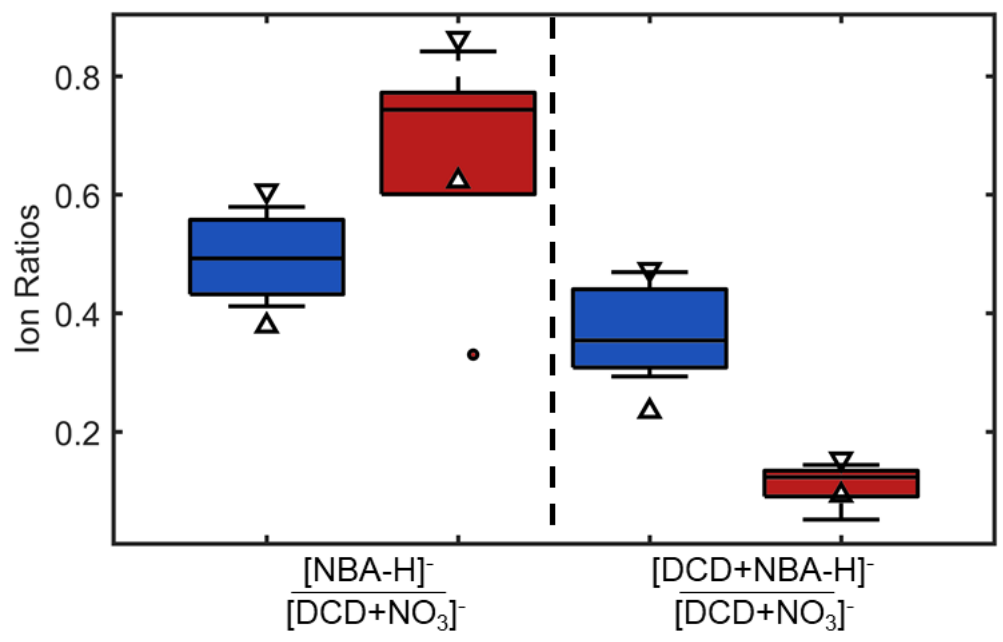

Figure S1. Box-whisker plots of ion ratios for solid (blue) and liquid (red) Triple Seven black powder substitute test samples. Boxes, whiskers, and outliers $(\bullet)$ signify median ion ratio with lower and upper quartiles, $1.5 \times$ interquartile range, and ratios beyond that range. Triangles represent the $95 \%$ confidence intervals around each distribution's median. 

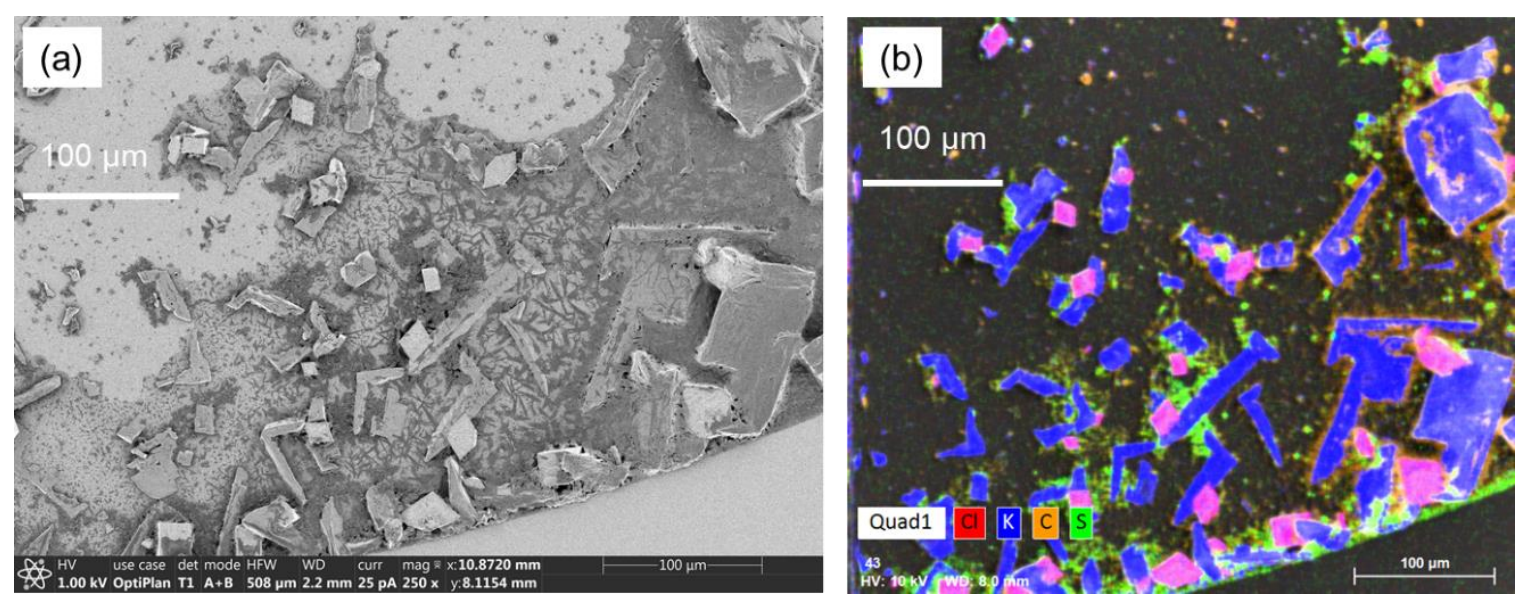

Figure S2. (a) SEM and (b) EDS images of solution cast Py rodex RS propellant. Mapped elements $\mathrm{Cl}, \mathrm{K}, \mathrm{C}$, and $\mathrm{S}$ displayed as red, blue, orange, and green. Scale bars represent $100 \mu \mathrm{m}$ in each image.

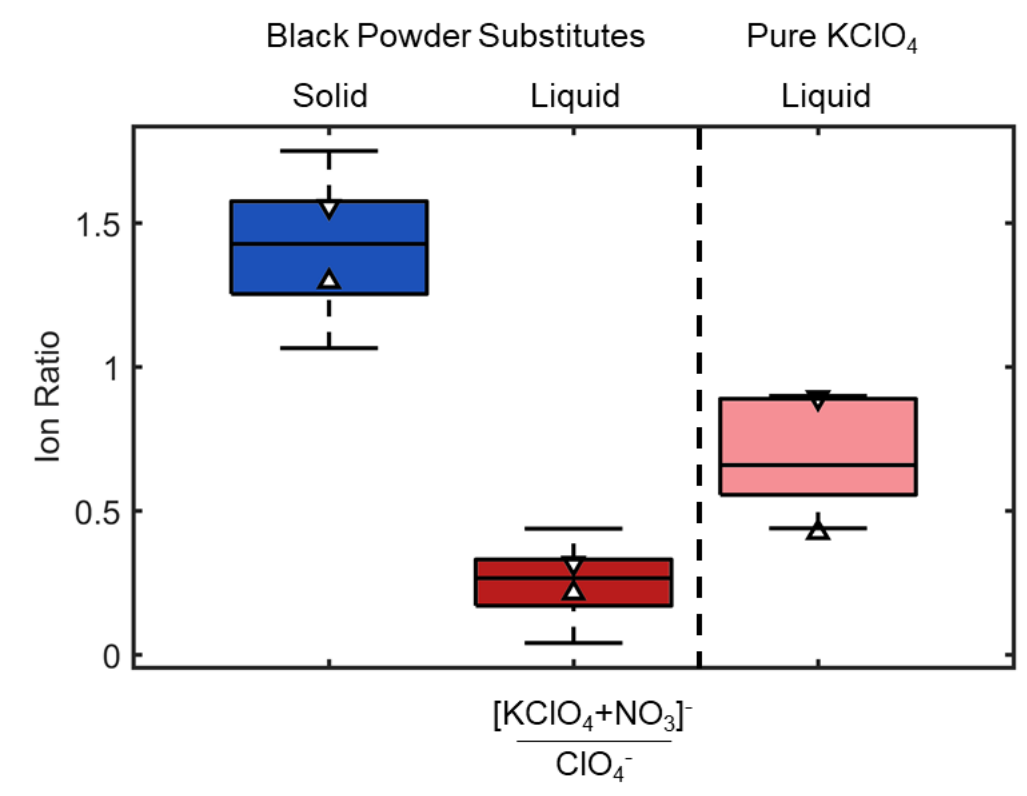

Figure S3. Differences in potassium perchlorate oxidizer ion ratios for solid (blue) and liquid (red) black powder substitute samples, and liquid (light red) pure potassium perchlorate. Triangles signify the $95 \%$ confidence intervals around each distribution's median. 

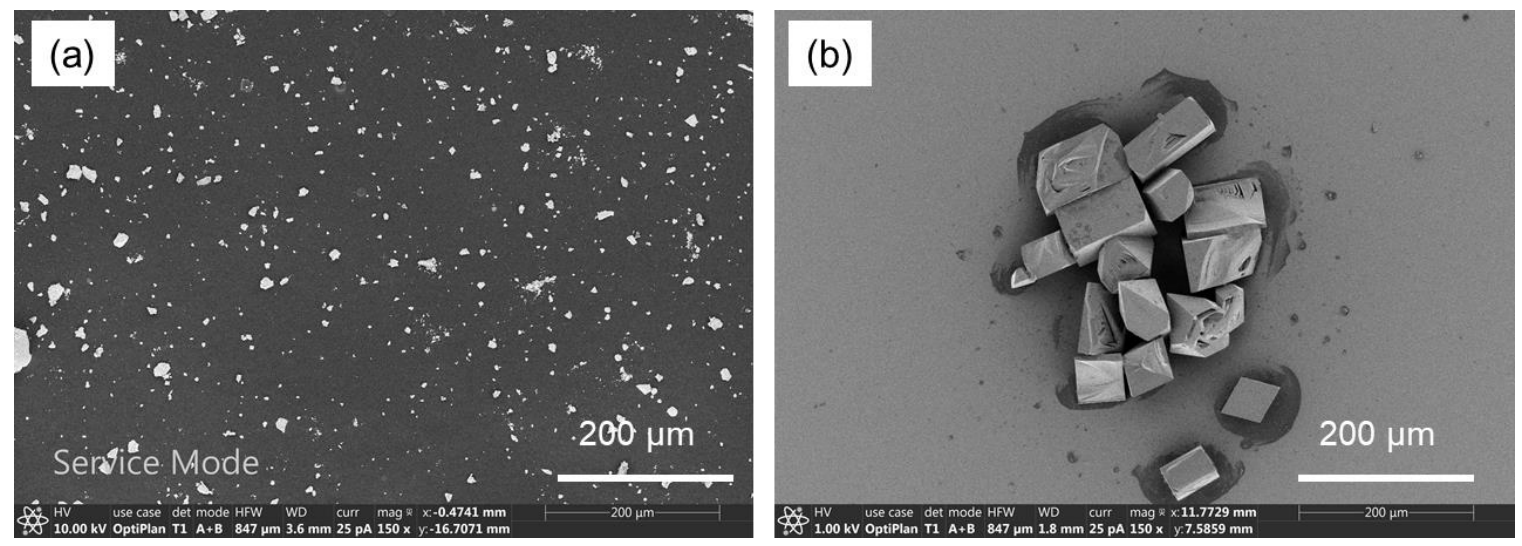

Figure S4. SEM images of (a) solid particle and (b) solution cast pure potassium perchlorate oxidizer. Scale bars represent $200 \mu \mathrm{m}$ in each image. 